\title{
3D Models as an Adjunct for Models in Studying Alzheimer's Disease
}

\author{
Ahmed Yaqinuddin ${ }^{1}$ Muhammad Faisal Ikram ${ }^{1}$ Ayesha Rahman Ambia ${ }^{1}$ Raghad Alaujan ${ }^{1}$ \\ Junaid Kashir ${ }^{1,2}$
}

${ }^{1}$ Department of Anatomy and Genetic, College of Medicine, Alfaisal University, Riyadh, Kingdom of Saudi Arabia

2 Department of Comparative Medicine, King Faisal Specialist Hospital and Research Center, Riyadh, Kingdom of Saudi Arabia

J Health Allied Sci ${ }^{\mathrm{NU}}$ 2022;12:179-185.

Address for correspondence Ahmed Yaqinuddin, MBBS, PhD, FRCP, College of Medicine, Riyadh 11533, Saudi Arabia

(e-mail: ayaqinuddin@alfaisal.edu).

\begin{abstract}
Keywords

- Alzheimer's disease

- animal models

- 3D culture

- stem cells
\end{abstract}

\section{Introduction}

First described in 1910 by Kraeplin, Alzheimer's disease (AD) is clinically and pathologically characteristic of dementia, defined as the progressive loss of two or more cognitive domains (i.e., memory, language, executive function, visuospatial function, personality, and behavior, loss of function and activities of daily living (ADL) ${ }^{1,2} \mathrm{AD}$ is the most common form of all dementias (80\%), affecting more than $30 \%$ of individuals of above 85 years. $^{2-4}$ Disease progression is marked by cognitive decline and memory impairment due to neurodegenerative processes in the brain stemming from amyloid- $\beta(A \beta)$ deposition and formation of neurofibrillary tangles. ${ }^{2-6}$ However, given the nature of the disease, it has been proven difficult to study the pathology and progression of this disease in vivo in humans. To this degree, AD models can help to study the disease pathogenesis, biochemistry, immunological functions, genetics, and potential pharmacotherapy. While animal and two-dimensional (2D) cell culture models have facilitated significant progress in studying the disease, more recent application of novel three-dimensional (3D) culture models has exhibited several advantages, which we aim to discuss in the current manuscript. ${ }^{7,8}$

\section{A Brief Profile of Alzheimer's Disease}

Pathogenesis in $\mathrm{AD}$ is dependent on two main neurological processes: formation of intracellular neurofibrillary tangles
DOI https://doi.org/ $10.1055 / \mathrm{s}-0041-1731864$. ISSN 2582-4287.

\footnotetext{
(c) 2021. Nitte (Deemed to be University). All rights reserved. This is an open access article published by Thieme under the terms of the Creative Commons Attribution-NonDerivative-NonCommercial-License, permitting copying and reproduction so long as the original work is given appropriate credit. Contents may not be used for commercial purposes, or adapted, remixed, transformed or built upon. (https://creativecommons.org/ licenses/by-nc-nd/4.0/)

Thieme Medical and Scientific Publishers Pvt. Ltd., A-12, 2nd Floor, Sector 2, Noida-201301 UP, India
} 
(NFTs) composed of hyperphosphorylated tau protein and deposition of extracellular senile $A \beta$ peptides. ${ }^{2}$ The tau protein produces tau-positive NFTs and neuropil, aggregated in neuronal and glial processes but lacks in cell bodies and starts forming initially in the medial transentorhinal region, eventually progressing to the neocortex. The presence of NFTs correlates with clinical severity, disease progression, and cognitive decline, unlike senile plaques, which initiates AD pathogenesis itself. AD is marked by loss of neurons, synapses, neurotransmitters, and neuroinflammation. ${ }^{9}$ The amyloid precursor protein (APP) gene, presenilin 1 (PSEN1) gene, and presenilin 2 (PSEN2) genes are responsible for the inherited forms of AD. APP is cleaved by the $\beta$-secretase and the $\gamma$-secretase complexes to form $A \beta$, whereas the PSEN1 and PSEN2 are components of the $\gamma$-secretase complex. The amyloid cascade hypothesis states that alterations in APP and $A B$ hypothesis leads to $A \beta$ protein aggregation and initiation of the cascade of $A D$ pathogenesis. An imbalance between $A \beta$ deposition and microglial clearance or local degradation determines the net amount of $A B$ in the brain. NFTs are not present in $\mathrm{AD}$ alone; they are correlated in at least twenty types of neurodegenerative disorders.

Tau is a hydrophilic protein, and is an important factor in microtubule assembly. Tau consists of large, unfolded regions encoded by the MAPT gene on chromosome 17 , consisting of four main protein domains. ${ }^{10}$ Mutation-induced alternative splicing affects the $\mathrm{N}$-terminal projection region and microtubule-binding domain, producing abnormal tau proteins and aggregation of tau protein into NFTs in a process termed taupathy. ${ }^{10}$ The accumulation of both $A B$ and NFTs proves to be neurotoxic, leading to neural degeneration. The clinical findings of AD can be variable, potentially progressing with the disease itself-mild cognitive impairment; loss of episodic memory or amnesia due to medial temporal lobe lesions; loss of semantic memory or knowledge (the general knowledge about facts and word meanings); and higher order cognitive functions such as language learning (e.g., aphasia) verbal fluency, problem-solving, or concept formation; and attention deficits in dual-processing tasks. ${ }^{1,2}$ There are also cases of visual and spatial disturbances, though these are less frequent. Definitive $\mathrm{AD}$ diagnosis involves postmortem study of brain morphology, cerebrospinal fluid (CSF) biomarkers, and positron emission tomography scans. ${ }^{1}$ Neuropsychiatric symptoms such as agitation, apathy, delusions, and disinhibition or problem behaviors such as resistance to care, obsessive-compulsive behaviors, caregiver shadowing, and hoarding behaviors can also manifest with the disease, and has to be managed by behavioral therapy. ${ }^{1,2,11}$

The risk factors for $A D$ include progressive age, inheritance of early-onset familial type $\mathrm{AD}$, traumatic brain injury, metal exposure, vascular injury, and infection. ${ }^{5}$ These risk factors modify the onset or/and pathogenesis of AD through the formation of oxygen-free radicals with increasing age; the consequence of "dual-hit hypothesis" of genetic predisposition and environmental stress; or the increase of "allostatic" load on body with time. Other risk factors include malnutrition, obesity, diabetes, mitochondrial dysfunction with maternal inheritance, immune system dysfunction, and psychiatric factors such as depression. ${ }^{5}$ The prevalence of AD is $19 \%$ in 7 to 84 years and between 30 to $35 \%$ and $50 \%$ for individuals above the age of 85 . Age is the most important risk factor for $\mathrm{AD}$, compared to other demographic data such as gender, race, ethnicity, or economic situation. ${ }^{5}$

Recent evidence indicates that immunological components of $\mathrm{AD}$ contribute to disease progression and severity. ${ }^{9}$ Microglial clearance of aggregated proteins or release of inflammatory cytokines due to microglial and astrocyte interaction with misfolded proteins via pattern-recognition receptors such as toll-like receptors scavenger receptors or receptors for advanced glycosylation end-products, suggest that neuroinflammation is significant in $\mathrm{AD}$ pathogenesis. ${ }^{6,9}$ Indeed, genes for immune receptors such as TREM2 and CD33 have been associated with AD. The microglia are resident phagocytes of the central nervous system that have physiological synapse-protective effects and immune clearance function for neuronal death and protein aggregation. Astrocytes and microglia also express APOE, and "activated" microglia expresses inflammatory markers such as $C D$ 36, CD14, CD11c, MHC-II, and iNOS. ${ }^{6,9}$ Microglial immunofactors induce an "A1-astrocyte" that contributes to neuronal death from decreased clearance, toxins release, and deficits in neurotropic factors. Microglial factors also induce shortening of the synaptic processes and swelling of the cell body. While acute activation of microglia may lead to increased phagocytic clearance of $A \beta$ aggregates, chronic inflammation leads to neurotoxicity and an inflammatory state. ${ }^{6,9}$

Three main genes have been identified that are responsible for familial types of AD (1-2\%), APP on chromosome 21, PSEN 1 on chromosome 14, and PSEN on chromosome 1 . A more prevalent form of $A D$ is the sporadic form, which is late onset compared to the familial type, and is associated with the APOE gene, an low-density lipoprotein cholesterol carrier. ${ }^{2,3}$ The type $\varepsilon 4$ allele for the APOE gene is present on chromosome 19 and is present in 50 to $60 \%$ of patients. ${ }^{3}$ The presence of one copy of this allele increases the risk of AD by threefold; two copies increase the risk eightfold. ${ }^{3}$ Genomewide studies have located 25 loci associated with late-onset $\mathrm{AD}$ now. Some genes that are associated with increased lateonset AD are ABCA7, CLU, CR1, CD33, CD2AP, EPHA1, BINN1, PICALM, and MS4A. ${ }^{3}$

$\mathrm{AD}$ is currently treated by cholinesterase inhibitors, with an addition of memantine for moderate-to-severe cases of $\mathrm{AD}$. The treatment is only symptomatic and does not reverse or prevent the pathology from occurring in high-risk patients. ${ }^{4,11}$ Although it does not change the disease course, cholinesterase inhibitors such as donepezil, rivastigmine, and galantamine are recommended at any stage of $\mathrm{AD}$. Memantine, a competitive N-methyl-D-aspartate antagonist and dopamine agonist have been approved for patients with a mini-mental state exam score $<15 .{ }^{4,11}$ Huperzine A has also been suggested as effective but is yet to receive approval from the Food and Drug Administration of United States. ${ }^{1,4}$ Omega-3 fatty acids from fish oil, for example, have also shown to improve recall and cognition. Vitamin D deficiency was also identified as an independent risk factor for the 
development of $\mathrm{AD}$; therefore, individuals with the vitamin deficiency are recommended to be prescribed supplements. The antioxidant properties of Vitamin $\mathrm{E}$ can also be used in AD without vascular risk factors. ${ }^{1}$ Lastly, management of cardiovascular risk factors through interventions such as physical exercise, especially regular aerobic exercise, and positive diets such as the Mediterranean diets have shown reduced burden of $\mathrm{AD}$ risk in studies. ${ }^{1}$

\section{Models Used in Alzheimer's Disease Study}

The criteria for an ideal model for disease study are recapitulating etiologies, presentation, and underlying pathology. Not only are models irreplaceable for study of disease mechanism and behavior, but they also provide insight into therapeutic advantages that can be harnessed. ${ }^{12}$ Most models are currently incapable of recapitulating complete disease characteristics of $\mathrm{AD}$, while even fewer models have the capacity of producing both amyloid plaques and NFTs, thereby enabling the study of the interplay between both pathological pathways with the other. Furthermore, it is impossible to predict downstream pathological artifacts or interaction between model endogenous molecular products; the strengths and weaknesses of all models can be studied and harnessed to reach a favorable end point in AD studies and therapeutic potentials. ${ }^{7}$

\section{Animal Models}

Animal models provide numerous ways to dissect the genetic, molecular, immunological, cellular, system and behavior, and therapeutic facets of most human diseases. Most commonly, rodent models are the primary animal model employed to study human AD, utilized for its genetic capacity through mouse genetic models. Previously, transgenic mice models had been utilized to study preclinical drug target models before conducting clinical trials. ${ }^{7,12,13}$ Transgenic rat models also exist, whose bigger brain size and similarity to human physiology and morphology allow for more sophisticated testing compared to transgenic mouse models. Transgenic rodent models, however, can only be utilized for familial AD and not the sporadic versions, which are instead studied through nonhuman primate models, whose larger brains and more CSF volume again pass for more sophisticated studies. Other physiological models used include dogs, guinea pigs, and the common degu. ${ }^{7}$ Drosophila melanogaster contains approximately $70 \%$ of human diseaserelated genes, and is also utilized to study neurodegenerative conditions including AD. Other animal models used include the invertebrate, nematode Caenorhabditis elegans, and vertebrate zebrafish, Danio rerio. ${ }^{13}$

Drosophila melanogaster expresses the APPL gene, which is a homolog of the APP gene. ${ }^{13}$ This APPL gene is processed by $\beta$-secretase enzyme, which releases the $A \beta$-like peptide. Transgenic manipulation of these flies can allow expression of the APP gene, produce enzymes to cleave APP into A $\beta$ peptides, or naturally express $A \beta$ peptides. Caenorhabditis elegans is an especially cheap, low-maintenance, and shortlifespan model, with $42 \%$ orthology with human disease genes. $^{13,14}$ Zebrafish are a fertile species that produce many transparent larvae and embryo at once and exhibit $70 \%$ of gene homology to humans. ${ }^{13,14}$ The extent of $A \beta$ neurotoxicity in zebrafish is, however, understudied. The effects of zebrafish with $A \beta$ peptide exposure in its aqueous environment have shown cognitive deterioration and avoidance of task performance. ${ }^{7,13}$ Early-onset or familial AD is a rarity $(<1 \%)$ caused due to autosomal dominant mutation in APP, PSEN1, or PSEN2. Most cases of AD are sporadic; this is not studied via rodent models. Transgenic rodent models mostly exhibit mutations in APP and PSEN1, possessing the greatest (97\%) sequence homology with human APP, with only differences in three amino acids that prevent the formation of amyloid plaques in mice models.

Amyloid plaques have been implicated to precede NFTs and appear in an independent fashion to the NFTs. ${ }^{7}$ Transgenic mice models form amyloid plaques through the expression of APP gene or with human PSEN1 and NFTs from expression of human MAPT. Mice models though have certain limitations. Despite having the most similarity with human genes among all animal models, it has a high failure rate in clinical trials (99.6\%) since these animals only mimic pathological manifestations in a nonphysiological manner for the ease of experimentation., ${ }^{72}$ Most of the disease-modifying therapies tested using conventional transgenic models had failed in phase 3. However, 3D models have largely replaced the difficulty in translation of results from animal models into clinical trials. The "amyloid hypothesis" states that $A \beta$ peptide aggregation from APP breakdown via $\beta$ - and $\gamma$-secretase causes plaque formation that sets off the accumulation of hyperphorylated tau protein and NFTs formation. ${ }^{14}$ The animal models also do not have the wide spectrum of other pathological features, most importantly the formation of the NFTs from hyperphosphorylated tau protein. Animal models have also posed the challenge of overexpression of APP, which can induce artificial pathologies. $^{7,12}$

\section{D Cultures}

Cell culture models utilize human tissue obtained through postmortem. As it is difficult to procure cells from quality tissue, obtaining cells of acceptable quality is supplanted via induced pluripotent stem cells (iPSCs). Multiple donor cells types such as fibroblasts, red blood cells, and epithelial cells can be utilized to give rise to stem cells. Recent developments in vitro have allowed for fibroblasts from patients with familial AD to generate iPSCs. ${ }^{15,16}$ The injection of pluripotency-genes such as OCT4, KLF4, SOX2, and c-MYC can induce fibroblasts to revert to their human embryonic stem cell stage, which can be differentiated into neurons, with identical genetic components of human AD. ${ }^{15,16}$ The cells are generated with a high level of purity, and closely resemble developmental timelines and signaling conditions-an ideal way to study developmental neurology. Stem cells are developed through a three-step procedure: neural induction, pattern formation, and terminal differentiation. After differentiating into a neuronal line, cells develop into cortical glutaminergic neurons through Wnt signaling and enhanced 
by sonic Hedgehog antagonists. ${ }^{15,16}$ External signaling is manipulated to differentiate other cell lines. Terminal differentiation of neuronal progenies then gives rise to mature neurons. ${ }^{15,16}$

A major drawbacks of employing cultures include the lack of protocol standardization, a simplified environment that do not represent the complex mechanisms ongoing in the brain, the requirement to generally age the pluripotent stem cells to develop the disease phenotype, and the knowledge that most cell cultures have been applied for the study of familial, rather than the sporadic form of AD. ${ }^{7,14,15,17}$ Another shortcoming of 2D models is the fact that they have been unable to show adequate levels of amyloid plaques, and $A \beta$ induced hyperphosphorylation and NFTs aggregation with paired helical filaments. ${ }^{14}$ This may be due to decreased production of $A \beta 42$ in $2 \mathrm{D}$ models compared to real patients, inadequate maturation of stem cells, and inability of simple 2D models to recapitulate a complex 3D brain environment. ${ }^{14}$ Stem cell lines are also homogeneous throughout the culture, and although they provide a high output for experimentation, they are not effective for studying the interplay between different cell lines. Such cells are also susceptible to genetic drift and can provide completely different phenotypes than the one desired for study. However, iPSC cultures have allowed the study of various human cell lines to study disease behavior, normal physiology, and conduct therapeutic trials. Clinical trials often fail in $\mathrm{AD}$ studies due to a lack of translation from rodent models to clinical trials. Stem cell cultures bypass this obstacle by allowing in-depth researches for both familial and sporadic types of the disease. ${ }^{16,18}$

\section{D Models}

3D models create a microfluid environment that recapitulates the environment seen in $\mathrm{AD}$ in humans to observe the interaction between neuronal cells, astrocytes, and microglia. By recapturing three important aspects of AD pathology, namely $A B$ aggregation, hyperphosphorylated tau aggregation, and the associated neuroinflammation, a triculture model of neurons, astrocytes, and microglia can be created. $3 \mathrm{D}$ culture models can be organoid, neurospheroid, or in a matrix. $^{8}$ The absence of the normal physiological brain environment in 2D cultures is compensated through the employment of 3D models that not only have the presence of other neuronal cells (e.g., glial cells) but also contain a scaffolding medium, such as hydrogel or Matrigel to allow a more physiological environment in the form of a 3D organoid. ${ }^{8,17,19}$ Furthermore, while 2D cultures exhibit $A \beta$ aggregate diffusion into the culture medium, 3D cultures can allow for concentration of these aggregates to form amyloid plaques. The third branch of the disease pathology, neuroinflammation, usually not recaptured in conventional models, is compensated by microglial secretion of proinflammatory markers secretion such as tumor necrosis factor-alpha, interferon gamma, and monocyte chemoattractant protein-1 in 3D models. The expression of neuronal and astrocyte markers, a higher surface area provided by the extracellular matrix (ECM) in 3D models, expression of latestage AD markers such as hyperphosphorylated tau formation, and study of gliosis, synaptic engulfment by microglial cells and neuronal loss are additional advantages of 3D models. $^{8,17,19}$

The $A B$ aggregation is seen in the early stages on $3 D$ culturing, that is, as early as 6 weeks. Hyperphosphorylated tau protein is seen in the late stages at around 10 to 14 weeks, a further advantage over 2D cultures and animal models, which do not express it. ${ }^{7,8,17,20}$ In designing 3D models, two major drivers are used: the use of human neural progenitor cells (hNPCs) in producing $A \beta$ aggregates and a Matrigel matrix that provides the ideal environment for hNPC growth and $A \beta$ aggregation. A hNPC cell line is first developed, immortalized, and infected with a lentivirus containing the APP and PSEN1 mutations through internal ribosomal entry sites. ${ }^{14,17}$ This is done to increase the $A B 42 / A \beta 40$ ratio as seen in $A D$. Induced pluripotent stem cells or iPSC from fibroblasts in the form of hNPC with the A246E mutation were created through genetic reprogramming using the lentivirus with Yamanaka factors such as Oct4, Klf4, Sox2, and c-Myc. These hNPCs produce the $A \beta$ oligers that contain the abnormal p21-activated kinase distribution as seen in $\mathrm{AD}$, which aggregate and kick off the disease biogenesis. ${ }^{21,22}$ Other studies have noted the use of hydrogels with hNPCs with mutated APP and PSEN1 gene, which expressed $A B$ on their own without the need for genetic reprogramming. ${ }^{22}$ The self-organizing behavior of $3 \mathrm{D}$ models using iPSCs with genetic mutation is utilized to create an AD phenotype with $A B$ aggregation and plaque formation. ${ }^{23} \mathrm{~A}$ fluorescence-activated cell sorting is then used to enrich cell populations. ${ }^{14,17}$ These differentiated cells are transferred to a Matrigel culture containing ECM brain proteins such as laminin, entactin, collagen, and heparin sulfate proteoglycans. ${ }^{14,17}$

Thin (100 to $300 \mu \mathrm{m}$ ) layer cultures for cellular imaging or thick $(\sim 4 \mathrm{~mm})$ layer cultures for biochemical and molecular studies can be synthesized depending on the goal of the experiment and can be used for small-scale studies or largescale drug testing. ${ }^{14,17}$ 3D brain models have already been employed to study the genetic and biochemical appliances of disorders such as microcephaly and autism. ${ }^{14,19}$ Not only have they been able to exhibit disease-specific phenotypes, but they can also be utilized for drug screening using highthroughput screening (HTS), RNA sequencing for transcriptome analysis, and genome-editing using CRISPR-Cas9, which can then be transplanted into the human brain. ${ }^{19}$ The self-organizing nature of brain 3D models is only held back by the lack of vascular and immunological structures that hinder the growth and offer a limitation for neurological disorders. They can be offset by newer vascularized organ buds or incorporation of microglia and vascular structures into organoids. One drawback of 3D models is that it does not represent specific brain locations such as the hippocampus or specific cortices. However, 3D models are relatively cost-effective and take shorter time (6-12 weeks) compared to animal models, which can take up to 2 to 3 years. Another excellent application is the HTS, which can screen thousands of chemical compounds in timeefficient manner. ${ }^{14,17,19}$ 


\section{Assimilating a 3D Culture Model}

The embedding of iPSCs in an ECM such as Matrigel, which is then put into a rotating bioreactor promotes proliferation and differentiation of neuronal tissue, instead of cells (as in 2D cultures), which is allowed to mature and develop synapses. Calcium activity is noticed in these organoids within 50 days from the start of culture. The current models of 3D organoids can be classified into a number of categories, such as sliced brain organoid culture, culture on microfluid chips, vascularized brain organoids, and specialized brain organoids. Growth factors, fibroblasts, and inflammatory cells have been also used to create a functional miniorgan comprising mature astrocytes, neuronal tissue, and progenitor cells. ${ }^{24}$ The process of assimilation of 3D cultures can be performed through various techniques. One such technique is the liquid overlay culture, where the culture surface is covered with Matrigel or agar or acarbose, and ECM is provided for spheroids to assemble when favorable conditions are achieved. ${ }^{25}$ One disadvantage is despite the controlled environment of the culture, the size of the spheroid is unpredictable and difficult to control. ${ }^{26} \mathrm{~A}$ hanging drop technique utilizes the technology of cell suspension of specific density and subsequent inversion to achieve a more controlled spheroid with regard to its volumetric dimensions. Hydrogels are a meshwork of highly water-soluble polymers intertwined with the procreant for tissue encapsulation. These with a soft-tissue consistency from collagen or alginate can be combined with basement membrane-like preparations. ${ }^{27}$ 3D bioreactors are largescale precise spheroid production machines through intermittent spinning or rotating of a cell suspension, with the technology for maintaining the nutrient supply and excrement removal. ${ }^{28}$ Scaffolds are made from various porous materials to mimic the ECM in vivo. They undergo fabrication processes, especially freeze-drying, where the water inside the polymer medium is sublimated, leading to pore formation. The only disadvantage here is the uncontrollability of pore size and biodegradability of the scaffold. ${ }^{29}$ 3D bioprinting prints solid materials that work as a novel application of tissue engineering, which are interconnected after the printing is completed. ${ }^{30}$

\section{Comparative Analysis: Advantages and Limitations of 3D Cultures versus Other Cultures}

The development of 3D models for studying brain organoids has been a great milestone in regenerative medicine through the use of iPSCs and embryonal cells to produce compartments or structures of brain organoids that are near-replicative of actual brain environment, such as the midbrain or hippocampus in vitro. ${ }^{24}$ The development of cutting-edge technologies such as gene sequencing and gene editing through CASPR/CAS9 has further implications in 3D spheroids applicability for studying diseases that were not possible to be studied in $2 \mathrm{D}$ cultures or animal models. The utilization of 3D cultures for making disease models and identifying drug target and evaluating therapeutic response in trial drugs has transformative potentials due to its cell-matrix interactions in addition to conventional cell-cell interactions. Not only that, advancements in controlled cell numbers, morphology, and protein synthesis have proven to be useful in many contemporary studies. $^{24,31}$ In comparison, 2D models with monolayer cells are economic, convenient, and are the most widely used cell cultures, though one major limitation that weakens its applicability is its inability to mimic physiological conditions in vivo, leading to results with limited application and therapeutic potential. This is due to its inability to mimic the cytoarchitecture and microenvironments in vivo. 2D models are still useful for obtaining quick preliminary results. $^{31,32}$ Animal models are instead time-consuming, expensive, and have certain moral dilemmas. 3D models also have greater representation of cell polarity and low stiffness. They also have greater heterogeneity in various stages that it can have at the same time-for example, proliferating or invasive or apoptotic. Although 3D cultures have their merits, some drawbacks of theirs is a lack of reproducibility and obscure study outcomes. ${ }^{33,34}$ Another limitation that 3D models has is the irregular distribution of growth media, which could show different outcomes in different regions of the models, or lead to heterogeneity in other terms. In comparison, 2D models have better reproducibility, but results achieved by these in vitro experiments fail to culminate in clinical trials. Also, the cell-cell interactions are less in 2D models compared to 3D models, and have been associated with further deviation from in vivo conditions. ${ }^{35}$

\section{Recent Studies Using 3D Models}

3D models have been applied in various fields to understand disease processes as in the case of cancers or the genetic mechanisms for expression patterns. A recent study on hepatocellular cancer, for example, noted enhanced control of tumor microenvironments and showed interestingly similar treatment response as real-life representation of the cancer, when compared to monolayer 2D models. Brain organoids have been utilized to study various regions of the brain, such as striatum, dorsal and ventral cortex, hippocampus and the choroid plexus, thalamus, hypothalamus, retina, midbrain, spinal cord, and the cerebellum. ${ }^{36}$ Today, the use of iPSCs in 3D cultures has led to far-reaching gains. They have been utilized in cancer research, stem cell research, drug trials, and disease-type studies. There have been a massive influx of researches on $3 \mathrm{D}$ cultures in the last decade, and there has also been indications that organoids can be possibly employed for organ transplantation. ${ }^{32}$ Tumor cells from patients can be harvested to study their disease and study individualized treatment responses. ${ }^{37}$ Stem cells used in these studies have investigated response to inflammation, hypoxia, angiogenesis, or stress, in these models. ${ }^{38} 3 \mathrm{D}$ models have also been applied for drug discovery, screening and testing, with results matching the clinical trials. ${ }^{37}$ 


\section{Ethical Concerns of 3D Models}

There are ethical concerns on the question of "developing consciousness" in brain organoids. The consciousness question is still debatable today, considering the electroencephalography (EEG) patterns resemble fetal brain activity at 16 to 21 weeks of intrauterine life; however, it should be noted that at this age of fetus consciousness is still not achieved. ${ }^{36}$

\section{Recommendations}

The cellular diversity, cytoarchitectural similarity, and environmental complexity of 3D models have been to produce physiological brain models that are uncontested by other conventional methods. The self-organizing behavior, cost and time-efficient, and ability to apply multimode studies for small- and large-scale studies have provided 3D models an indisputable advantage over animal or iPSC culture models. Moreover, 3D models not only encompass the benefits of rodent models and 2D models in studying disease genomics, biochemistry, and molecular mechanisms but also surpass milestones not covered by their precedents: the formation of hyperphosphorylated tau protein and NFTs to study the latestages of $A D$, which were not possible in animal models or cell cultures. Of the three in vitro studies, 3D models are the most representative of the physiologic brain environment, allowing for an in-depth study of the disease and develop therapeutic breakthroughs, which had mostly failed through rodent model translation into clinical trials. 3D model organoids can also be utilized for genetic editing through CASPR/cas9, which can be used for in vivo transplantation. With the above-mentioned strengths, it is inevitably conspicuous that 3D models are going to be the powerhouse for studying many neurological diseases and will make record breakthroughs with further application of different study models using 3D organoids.

\section{Conflict of Interest}

None declared.

\section{References}

1 Atri A. The Alzheimer's disease clinical spectrum: diagnosis and management. Med Clin North Am 2019;103(02):263-293

2 Bondi MW, Edmonds EC, Salmon DP. Alzheimer's disease: past, present, and future. J Int Neuropsychol Soc 2017;23(9-10) 818-831

3 Karch CM, Goate AM. Alzheimer's disease risk genes and mechanisms of disease pathogenesis. Biol Psychiatry 2015;77(01): 43-51

4 Weller J, Budson A. Current understanding of Alzheimer's disease diagnosis and treatment. F1000 Res 2018;7:7

5 Armstrong R. Risk factors for Alzheimer's disease. Folia Neuropathol 2019;57(02):87-105

6 Sarlus H, Heneka MT. Microglia in Alzheimer's disease. J Clin Invest 2017;127(09):3240-3249

7 Drummond E, Wisniewski T. Alzheimer's disease: experimental models and reality. Acta Neuropathol 2017;133(02):155-175

8 Park J, Wetzel I, Marriott Iet al.A 3D human triculture system modeling neurodegeneration and neuroinflammation in Alzheimer's disease. Nat Neurosci 2018;21(07):941-951
9 Heneka MT, Carson MJ, El Khoury Jet al.Neuroinflammation in Alzheimer's disease. Lancet Neurol 2015;14(04):388-405

10 Naseri NN, Wang H, Guo J, Sharma M, Luo W. The complexity of tau in Alzheimer's disease. Neurosci Lett 2019;705:183-194

11 Chu LW. Alzheimer's disease: early diagnosis and treatment. Hong Kong Med J 2012;18(03):228-237

12 Nazem A, Sankowski R, Bacher M, Al-Abed Y. Rodent models of neuroinflammation for Alzheimer's disease. J Neuroinflammation 2015;12:74

13 Vanderheyden WM, Lim MM, Musiek ES, Gerstner JR. Alzheimer's disease and sleep-Wake disturbances: amyloid, astrocytes, and animal models. J Neurosci 2018;38(12):2901-2910

14 Choi SH, Kim YH, Quinti L, Tanzi RE, Kim DY. 3D culture models of Alzheimer's disease: a road map to a "cure-in-a-dish". Mol Neurodegener 2016;11(01):75

15 Arber C, Lovejoy C, Wray S. Stem cell models of Alzheimer's disease: progress and challenges. Alzheimers Res Ther 2017;9 (01):42

16 Mungenast AE, Siegert S, Tsai LH. Modeling Alzheimer's disease with human induced pluripotent stem (iPS) cells. Mol Cell Neurosci 2016;73:13-31

17 Kim YH, Choi SH, D’Avanzo Cet al.A 3D human neural cell culture system for modeling Alzheimer's disease. Nat Protoc 2015;10 (07):985-1006

18 Stansley B, Post J, Hensley K. A comparative review of cell culture systems for the study of microglial biology in Alzheimer's disease. J Neuroinflammation 2012;9:115

19 Lee CT, Bendriem RM, Wu WW, Shen RF. 3D brain Organoids derived from pluripotent stem cells: promising experimental models for brain development and neurodegenerative disorders. J Biomed Sci 2017;24(01):59

20 Cenini G, Hebisch M, Iefremova Vet al.Dissecting Alzheimer's disease pathogenesis in human 2D and 3D models. Mol Cell Neurosci 2021;110:103568

21 Hernández-Sapiéns MA, Reza-Zaldívar EE, Cevallos RR, MárquezAguirre AL, Gazarian K, Canales-Aguirre AA. A three-dimensional Alzheimer's disease cell culture model using iPSC-derived neurons carrying A246E mutation in PSEN1. Front Cell Neurosci 2020; $14: 151$

22 Zhang D, Pekkanen-Mattila M, Shahsavani M, Falk A, Teixeira AI, Herland A. A 3D Alzheimer's disease culture model and the induction of P21-activated kinase mediated sensing in iPSC derived neurons. Biomaterials 2014;35(05):1420-1428

23 Raja WK, Mungenast AE, Lin YTet al.Self-organizing 3d human neural tissue derived from induced pluripotent stem cells recapitulate Alzheimer's disease phenotypes. PLoS One 2016;11(09): 0161969

24 Shou Y, Liang F, Xu S, Li X. The application of brain organoids: from neuronal development to neurological diseases. Front Cell Dev Biol 2020;8:579659

25 Vinci M, Gowan S, Boxall Fet al.Advances in establishment and analysis of three-dimensional tumor spheroid-based functional assays for target validation and drug evaluation. BMC Biol 2012; $10: 29$

26 Kelm JM, Timmins NE, Brown CJ, Fussenegger M, Nielsen LK. Method for generation of homogeneous multicellular tumor spheroids applicable to a wide variety of cell types. Biotechnol Bioeng 2003;83(02):173-180

27 Drury JL, Mooney DJ. Hydrogels for tissue engineering: scaffold design variables and applications. Biomaterials 2003;24(24): 4337-4351

28 Rauh J, Milan F, Günther KP, Stiehler M. Bioreactor systems for bone tissue engineering. Tissue Eng Part B Rev 2011;17(04): 263-280

29 Chen CY, Ke CJ, Yen KC, Hsieh HC, Sun JS, Lin FH. 3D porous calcium-alginate scaffolds cell culture system improved human osteoblast cell clusters for cell therapy. Theranostics 2015;5(06): 643-655 
30 Dai X, Ma C, Lan Q, Xu T. 3D bioprinted glioma stem cells for brain tumor model and applications of drug susceptibility. Biofabrication 2016;8(04):045005

31 Chaicharoenaudomrung N, Kunhorm P, Noisa P. Three-dimensional cell culture systems as an in vitro platform for cancer and stem cell modeling. World J Stem Cells 2019;11(12):1065-1083

32 Jensen $C$, Teng Y. Is it time to start transitioning from 2D to 3D cell culture? Front Mol Biosci 2020;7:33

33 Hickman JA, Graeser R, de Hoogt R, et al.IMI PREDECT Consortium. Three-dimensional models of cancer for pharmacology and cancer cell biology: capturing tumor complexity in vitro/ex vivo. Biotechnol J 2014;9(09):1115-1128

34 Ravi M, Paramesh V, Kaviya SR, Anuradha E, Solomon FD. 3D cell culture systems: advantages and applications. J Cell Physiol 2015; 230(01):16-26
35 Bordanaba-Florit G, Madarieta I, Olalde B, Falcón-Pérez JM, Royo F. 3D cell cultures as prospective models to study extracellular vesicles in cancer. Cancers (Basel) 2021;13(02):307

36 Tanaka Y, Park IH. Regional specification and complementation with non-neuroectodermal cells in human brain organoids. J Mol Med (Berl) 2021;99(04):489-500

37 Langhans SA. Three-dimensional in vitro cell culture models in drug discovery and drug repositioning. Front Pharmacol 2018;9:6

38 Potapova IA, Gaudette GR, Brink PRet al.Mesenchymal stem cells support migration, extracellular matrix invasion, proliferation, and survival of endothelial cells in vitro. Stem Cells 2007;25(07): $1761-1768$ 